\title{
PRINCIPAL MATRIX SOLUTIONS AND VARIATION OF PARAMETERS FOR VOLTERRA INTEGRO-DYNAMIC EQUATIONS ON TIME SCALES
}

\author{
MURAT ADIVAR \\ Department of Mathematics, Izmir University of Economics \\ 35330, Balcova, Izmir, Turkey \\ e-mail:murat.adivar@ieu.edu.tr
}

(Received 8 February 2010; revised 5 May 2010; accepted 21 September 2010; first published online 10 March 2011)

Abstract. We introduce the principal matrix solution $Z(t, s)$ of the linear Volterra-
type vector integro-dynamic equation
\[ x^{\Delta}(t)=A(t) x(t)+\int_{s}^{t} B(t, u) x(u) \Delta u \]

and prove that it is the unique matrix solution of

$$
Z^{\Delta_{t}}(t, s)=A(t) Z(t, s)+\int_{s}^{t} B(t, u) Z(u, s) \Delta u, \quad Z(s, s)=I .
$$

We also show that the solution of

$$
x^{\Delta}(t)=A(t) x(t)+\int_{\tau}^{t} B(t, u) x(u) \Delta u+f(t), \quad x(\tau)=x_{0}
$$

is unique and given by the variation of parameters formula

$$
x(t)=Z(t, \tau) x_{0}+\int_{\tau}^{t} Z(t, \sigma(s)) f(s) \Delta s .
$$

2010 Mathematics Subject Classification. 34N05, 45D05, 39A13, 45J05.

1. Introduction and preliminaries. The resolvent equation and variation of parameters formula play a key role in the stability analysis of Volterra-type integral (or summation) equations (see $[\mathbf{6}, \mathbf{9}, \mathbf{1 0}, \mathbf{1 5}]$, and references therein). The investigation of Volterra-type integral equations with the aid of resolvent was first begun by Grossmann and Miller [13] in their outstanding study. Grossmann and Miller [13] defined the resolvent $R(t, s)$ by

$$
R(t, s)=I+\int_{s}^{t} R(t, u) \Psi(u, s) d u \quad 0 \leq s \leq t,
$$

where $I$ is the identity matrix and

$$
\Psi(t, s)=A(t)+\int_{s}^{t} B(t, v) d v
$$


and showed that Volterra vector integro-differential equation

$$
x^{\prime}(t)=A(t) x(t)+\int_{0}^{t} B(t, u) x(u) d u+f(t)
$$

has the unique continuous solution

$$
x(t)=R(t, 0) x_{0}+\int_{0}^{t} R(t, s) f(s) d s
$$

satisfying the initial condition $x(0)=0$. They also proved that $R(t, s)$ is continuous for $0 \leq s \leq t$ and satisfies the resolvent equation

$$
\frac{\partial}{\partial s} R(t, s)=-R(t, s) A(s)-\int_{s}^{t} R(t, u) B(u, s) d u, \quad R(t, t)=I .
$$

In a discrete case, Elaydi [9] obtained the resolvent equation as follows:

$$
R(n+1, m)=\tilde{A}(n) R(n, m)+\sum_{k=m}^{n} B(n, k) R(k, m), \quad n \geq m,
$$

with $R(m, m)=I$. Using (3), Elaydi derived the variation of parameters formula

$$
y\left(n, 0, y_{0}\right)=R(n, 0) y_{0}+\sum_{k=0}^{n-1} R(n, k+1) g(k)
$$

for the unique solution $y\left(n, 0, y_{0}\right)$ of the equation

$$
y(n+1)=\tilde{A}(n) y(n)+\sum_{k=0}^{n} B(n, k) y(k)+g(k)
$$

satisfying $y(0)=y_{0}($ see [9, Proposition 1]). Recently, Eloe et al. [11] arrived at the resolvent equation

$$
\Delta_{m} R(n, m)=-R(n, m+1)(\tilde{A}(m)-I)-\sum_{k=m}^{n-1} R(n, k+1) B(k, m),
$$

for $m \leq n$, with $R(m, m)=I$ and $R(n, m)=0$ if $n<m$, where $\Delta_{m} R(n, m)=R(n, m+$ $1)-R(n, m)$. It is worth mentioning that equation (5) is the discrete analogue of equation (2) with $A(m)=\tilde{A}(m)-I$.

Afterwards, Becker [2] found a more fundamental way of defining resolvent and variation of parameters formula for the unique solution of (1) satisfying $x(0)=x_{0}$. He introduced the principal matrix solution $Z(t, s)$ of the homogeneous Volterra equation

$$
x^{\prime}(t)=A(t) x(t)+\int_{0}^{t} B(t, u) x(u) d u
$$

to derive the variation of parameters formula

$$
x(t)=Z(t, 0) x_{0}+\int_{0}^{t} Z(t, s) f(s) d s
$$


and showed that $Z(t, s)$ satisfies

$$
\frac{\partial}{\partial t} Z(t, s)=A(t) Z(t, s)+\int_{s}^{t} B(t, u) Z(u, s) d u, \quad Z(s, s)=I,
$$

which is the principal matrix version of the resolvent equation (2). Becker also proved that Miller's resolvent $R(t, s)$ is the transpose of the principal matrix solution of the adjoint equation

$$
y^{\prime}(s)=-A^{T}(s) y(s)-\int_{s}^{t} B^{T}(u, s) y(u) d u
$$

and $R(t, s)$ and $Z(t, s)$ are identical despite the difference between their definitions. Note that Becker's remarkable resolvent $Z(t, s)$ took a prominent attention in recent years (see, for instance, $[\mathbf{6 - 1 0}, \mathbf{1 8}, \mathbf{2 0}]$ ).

During last decades, theory of time scales became a useful device for the unification of differential and difference equations under dynamic equations on time scales (see $[4,5]$ and references therein). In order to indicate a time scale (a closed non-empty subset of reals) we use the notation $\mathbb{T}$. We classify the points of a time scale $\mathbb{T}$ by using the forward jump and backward jump operators defined by

$$
\sigma(t):=\inf \{s \in \mathbb{T}: s>t\}
$$

and

$$
\rho(t):=\sup \{s \in \mathbb{T}: s<t\}
$$

respectively. A point $t$ in $\mathbb{T}$ is said to be right-scattered (right-dense) if $\sigma(t)>t(\sigma(t)=t)$. We say $t \in \mathbb{T}$ is left-scattered (left-dense) if $\rho(t)<t(\rho(t)=t)$. If $\rho(t)<t<\sigma(t)$, then $t \in \mathbb{T}$ is called isolated point. The set $\mathbb{T}^{\kappa}$ is derived from the time scale $\mathbb{T}$ as follows: If $\mathbb{T}$ has a left-scattered maximum $m$, then $\mathbb{T}^{\kappa}=\mathbb{T}-\{m\}$. Otherwise $\mathbb{T}^{\kappa}=\mathbb{T}$. The delta derivative of a function $f: \mathbb{T} \rightarrow \mathbb{R}$, defined at a point $t \in \mathbb{T}^{\kappa}$ by

$$
f^{\Delta}(t):=\lim _{\substack{s \rightarrow t \\ s \neq \sigma(t)}} \frac{f(\sigma(t))-f(s)}{\sigma(t)-s}
$$

was first introduced by Hilger [14] to unify discrete and continuous analyses. It follows from definition of the operator $\sigma$ that

$$
\sigma(t)=\left\{\begin{array}{cl}
t & \text { if } \mathbb{T}=\mathbb{R} \\
t+1 & \text { if } \mathbb{\mathbb { T }}=\mathbb{Z} \\
q t & \text { if } \mathbb{\mathbb { T }}=\overline{q^{\mathbb{Z}}}
\end{array}\right.
$$

where $\overline{q^{\mathbb{Z}}}=\left\{q^{k}: k \in \mathbb{Z}\right\} \cup\{0\}$ and $q>1$. Hence, the delta derivative $f^{\Delta}(t)$ turns into ordinary derivative $f^{\prime}(t)$ if $\mathbb{T}=\mathbb{R}$ and it becomes the forward difference operator $\Delta f(t):=f(t+1)-f(t)$ whenever $\mathbb{T}=\mathbb{Z}$. For the time scale $\mathbb{T}=\overline{q^{\mathbb{Z}}}$ we have $f^{\Delta}(t)=$ $D_{q} f(t)$, where

$$
D_{q} f(t):=\frac{f(q t)-f(t)}{(q-1) t}
$$


Thus, one can consider the differential, difference and $q$-difference equations as special cases of the dynamic equations on time scales. It is evident from the recent literature $($ see $[1,3,4,5,16,17,19])$ that dynamic equations on time scales are a useful tool to explain the differences and similarities between difference and differential equations. It turns out that the theory of time scales provide a deep understanding of physical application and leads to the invention of some results that are not known even for the special cases of time scales. Furthermore, since there are many time scales that are different than $\mathbb{R}, \mathbb{Z}$ and $\overline{q^{\mathbb{Z}}}$, investigation of dynamic equations on time scales provides a more general and comprehensive theory.

Hereafter, we denote by $[a, b]_{\mathbb{T}}$ the closed interval $[a, b] \cap \mathbb{\mathbb { V }}$ of the time scale $\mathbb{T}$. The intervals $[a, b)_{\mathbb{T}},(a, b]_{\mathbb{T}}$ and $(a, b)_{\mathbb{\mathbb { T }}}$ are defined similarly. A function $f: \mathbb{T} \rightarrow \mathbb{R}$ is called $r d$-continuous if it is continuous at right dense points and its left-sided limits exist (finite) at left dense points. The set of $r d$-continuous functions $f: \mathbb{T} \rightarrow \mathbb{R}$ is denoted by $C_{r d}=C_{r d}(\mathbb{T})$. Note that continuity implies $r d$-continuity. Every $r d$-continuous function $f: \mathbb{T} \rightarrow \mathbb{R}$ has an anti-derivative

$$
F(t)=\int_{t_{0}}^{t} f(t) \Delta t
$$

That is, $F^{\Delta}(t)=f(t)$ for all $t \in \mathbb{T}^{\kappa}$ (see [5, Theorem 1.74]). For a comprehensive review on $\Delta$-derivative and $\Delta$-Riemann integral we direct the reader to [4].

Let $a, b \in \mathbb{T}$ with $a<b$ and $f \in C_{r d}$. We know from [5, Theorem 1.79] that

$$
\begin{gathered}
\int_{a}^{b} f(t) \Delta t=\int_{a}^{b} f(t) d t \text { if } \mathbb{T}=\mathbb{R}, \\
\int_{a}^{b} f(t) \Delta t=\sum_{t=a}^{b-1} f(t) \text { if } \mathbb{T}=\mathbb{Z}
\end{gathered}
$$

and

$$
\int_{a}^{b} f(t) \Delta t=(q-1) \sum_{t \in[a, b) \cap \overline{q^{\mathbb{Z}}}} t f(t) \text { if } \mathbb{\mathbb { Z }}=\overline{q^{\mathbb{Z}}}
$$

Hence, integral, summation and $q$-integral equations can be regarded as special cases of integral equations on time scales.

The study in [1] is the first of its kind in which the resolvent concept has been investigated on time scales. In [1], the authors showed that a solution of the linear system of integral equations

$$
x(t)=f(t)+\int_{t_{0}}^{t} a(t, s) x(s) \Delta s, \quad t_{0} \in \mathbb{T}^{\kappa}
$$

can be given by the variation of parameters formula

$$
x(t)=f(t)-\int_{t_{0}}^{t} r(t, u) f(u) \Delta u
$$


where the resolvent $r(t, s)$ is the unique solution of

$$
r(t, s)=-a(t, s)+\int_{\sigma(s)}^{t} r(t, u) a(u, s) \Delta u .
$$

If $a$ is scalar-valued, so is $r$. If $a$ is $n \times n$ matrix, so is $r$. However, there is no variation of parameters formula for the solutions of integro-dynamic equation

$$
x^{\Delta}(t)=A(t) x(t)+\int_{\tau}^{t} B(t, u) x(u) \Delta u+f(t), t \in\left[t_{0}, \infty\right)_{\mathbb{T} \kappa},
$$

where $\tau \in\left[t_{0}, \infty\right)_{\mathbb{\pi}^{\kappa}}$ is fixed, $A$ is an $n \times n$ matrix function that is continuous on $\left[t_{0}, \infty\right)_{\mathbb{J}^{\kappa}}$ and $B$ is an $n \times n$ matrix function that is continuous on

$$
\Omega:=\left\{(t, u) \in \mathbb{T}^{\kappa} \times \mathbb{T}^{\kappa}: t_{0} \leq u \leq t<\infty\right\} .
$$

This work is the continuation of the paper [1] and aims to achieve the following three main tasks:

- To show the existence and uniqueness of the continuous solution of (10) satisfying $x(\tau)=x_{0}$.

- To introduce the principal matrix solution $Z(t, s)$ of the integro-dynamic equation

$$
x^{\Delta}(t)=A(t) x(t)+\int_{s}^{t} B(t, u) x(u) \Delta u .
$$

- To derive the variation of parameters formula

$$
x(t)=Z(t, \tau) x_{0}+\int_{\tau}^{t} Z(t, \sigma(s)) f(s) \Delta s
$$

for the unique solution of $x(t)$ of equation (10) satisfying $x(\tau)=x_{0}$.

Hence, the theory developed in [2] is extended to general time scales.

Notice that for the particular cases $\mathbb{T}=\mathbb{R}, \mathbb{T}=\mathbb{Z}$ and $\mathbb{T}=\overline{q^{\mathbb{Z}}}$, the Volterra integrodynamic equation (10) turns into Volterra vector integro-differential equation (1), Volterra-type integro-difference equation (4) and Volterra-type integro $q$-difference equation

$$
D_{q} x(t)=A(t) x(t)+\sum_{s \in[\tau, t) \cap q^{\mathbb{Z}}} \tilde{B}(t, u) x(u)+f(t),
$$

respectively, where $\tilde{B}(t, u):=u(q-1) B(t, u)$ and $D_{q}$ is the $q$-difference operator defined by (6). Since there are more time scales other than $\mathbb{T}=\mathbb{R}, \mathbb{T}=\mathbb{Z}$ and $\mathbb{T}=\overline{q^{\mathbb{Z}} \text {, }}$ investigation of resolvent and principal matrix solution on time scales provides a more general theory. Moreover, some results obtained in this paper are new for difference and $q$-difference equations.

For brevity we assume familiarity with time scale calculus. For an excellent review on time scales we direct the reader to $[\mathbf{4}, \mathbf{5}]$. We end this section by listing some results, definitions and notations from the existing literature for further use.

Throughout the paper we denote by $\mu$ the graininess (step-size) function defined by $\mu(t):=\sigma(t)-t$. 
In order to construct the Banach space $C[a, b]_{\mathbb{T}}$ used in further sections we need to define the exponential function $e_{\alpha}(t, s)$, which is the unique solution of the initial value problem $y^{\Delta}=\alpha(t) y, y(s)=1$ provided $\alpha$ is regressive (see [4, Theorem 2.35]).

Definition 1. A function $\alpha: \mathbb{T} \rightarrow \mathbb{R}$ is said to be regressive provided $1+$ $\mu(t) \alpha(t) \neq 0$ for all $t \in \mathbb{T}^{\kappa}$. The set of all regressive rd-continuous functions $\alpha: \mathbb{T} \rightarrow \mathbb{R}$ is denoted by $\mathcal{R}$, while the set $\mathcal{R}^{+}$is given by $\mathcal{R}^{+}=\{\alpha \in \mathcal{R}: 1+\mu(t) \alpha(t)>0$ for all $t \in$ ए\}.

Let $\alpha \in \mathcal{R}$ and $\mu(t)>0$ for all $t \in \mathbb{T}$. The exponential function on $\mathbb{\mathbb { T }}$ is defined by

$$
e_{\alpha}(t, s):=\exp \left(\int_{s}^{t} \frac{1}{\mu(z)} \log (1+\mu(z) \alpha(z)) \Delta z\right) .
$$

It is well known that if $p \in \mathcal{R}^{+}$, then $e_{p}(t, s)>0$ for all $t \in \mathbb{T}$. Other properties of the exponential function are given in the following lemma.

Lemma 2. [4, Theorem 2.36]. Let $p, q \in \mathcal{R}$. Then

(i) $e_{0}(t, s) \equiv 1$ and $e_{p}(t, t) \equiv 1$;

(ii) $e_{p}(\sigma(t), s)=(1+\mu(t) p(t)) e_{p}(t, s)$;

(iii) $\frac{1}{e_{p}(t, s)}=e_{\ominus p}(t, s)$ where, $\ominus p(t)=-\frac{p(t)}{1+\mu(t) p(t)}$;

(iv) $e_{p}(t, s)=\frac{1}{e_{p}(s, t)}=e_{\ominus p}(s, t)$;

(v) $e_{p}(t, s) e_{p}(s, r)=e_{p}(t, r)$;

(vi) $\left(\frac{1}{e_{p}(\cdot, s)}\right)^{\Delta}=-\frac{p(t)}{e_{p}^{\sigma}(\cdot, s)}$.

Lemma 3. [3, Remarks 1-2].

(i) For a non-negative $\varphi$ with $-\varphi \in \mathcal{R}^{+}$, we have the inequalities

$$
1-\int_{s}^{t} \varphi(u) \Delta u \leq e_{-\varphi}(t, s) \leq \exp \left\{-\int_{s}^{t} \varphi(u) \Delta u\right\} \text { for all } t \geq s .
$$

(ii) If $\varphi$ is rd-continuous and non-negative, then

$$
1+\int_{s}^{t} \varphi(u) \Delta u \leq e_{\varphi}(t, s) \leq \exp \left\{\int_{s}^{t} \varphi(u) \Delta u\right\} \text { for all } t \geq s .
$$

TheOREM 4. [4, Theorem 1.117]. Let $a \in \mathbb{T}^{\kappa}, b \in \mathbb{T}$ and assume that $k: \mathbb{T} \times \mathbb{T}^{\kappa} \rightarrow \mathbb{R}$ is continuous at $(t, t)$, where $t \in \mathbb{T}^{\kappa}$ with $t>a$. Also assume that $k^{\Delta}(t,$.$) is r d$-continuous on $[a, \sigma(t)]$. Suppose that for each $\varepsilon>0$ there exists a neighbourhood $U$ of $t$, independent of $\tau \in[a, \sigma(t)]$, such that

$$
\left|k(\sigma(t), \tau)-k(s, \tau)-k^{\Delta}(t, \tau)(\sigma(t)-s)\right| \leq \varepsilon|\sigma(t)-s|
$$

for all $s \in U$, where $k^{\Delta}$ denotes the derivative of $k$ with respect to the first variable. Then

$$
\begin{aligned}
g(t) & :=\int_{a}^{t} k(t, \tau) \Delta \tau \text { implies } g^{\Delta}(t)=\int_{a}^{t} k^{\Delta}(t, \tau) \Delta \tau+k(\sigma(t), t), \\
h(t) & :=\int_{t}^{b} k(t, \tau) \Delta \tau \text { implies } g^{\Delta}(t)=\int_{t}^{b} k^{\Delta}(t, \tau) \Delta \tau-k(\sigma(t), t) .
\end{aligned}
$$


Lemma 5. (Gronwall's inequality) [4, Corollary 6.7]. Let $y \in C_{r d}, \alpha \in \mathcal{R}^{+}$and $c \in \mathbb{R}$. Then

$$
y(t) \leq c+\int_{t_{0}}^{t} y(s) \alpha(s) \Delta s \text { for all } t \in \mathbb{T}
$$

implies

$$
y(t) \leq c e_{\alpha}\left(t, t_{0}\right) \text { for all } t \in \mathbb{T} .
$$

THEOREM 6. [12, Theorem 5, p. 102]. Let $f(x, y)$ be a real-finite valued function whose domain is the Cartesian product $S_{1} \times S_{2}$. Suppose $f(x, y)$ is continuous in $y$ at $y=b$ uniformly for $x$ in $S_{1}$, and continuous in $x$ at $x=$ a for each $y$ in $S_{2}$, then $f(x, y)$ is continuous in $(x, y)$ at $(a, b)$.

2. Existence and Uniqueness. In this section, we use the Banach contraction mapping principle to show the existence of a unique continuous solution of

$$
x^{\Delta}(t)=A(t) x(t)+\int_{s}^{t} B(t, u) x(u) \Delta u+f(t), t \in[s, \infty)_{\llbracket k}
$$

satisfying $x(s)=x_{0}$, where $s \in \mathbb{T}^{\kappa}$ is fixed, $A$ is an $n \times n$ matrix function that is continuous on $\left[t_{0}, \infty\right)_{\mathbb{\pi}^{\kappa}}, B$ is an $n \times n$ matrix function that is continuous on

$$
\Omega:=\left\{(t, s) \in \mathbb{T}^{\kappa} \times \mathbb{T}^{\kappa}: t_{0} \leq s \leq t<\infty\right\}
$$

and $f$ is $n$-vector function that is continuous on $\left[t_{0}, \infty\right)_{\mathbb{T}}$. For this purpose, we first introduce the Banach space in which the equation (11) will be proven to have a solution.

2.1. Banach space $\left(\mathbf{C}[a, b]_{\mathbb{T}}, d_{\beta}\right)$. Denote by $\|$.$\| the vector norm on \mathbb{R}^{n}$. For an $n \times n$ matrix $A$ we define the matrix norm $|A|$ induced by the vector norm by

$$
|A|:=\sup \{\|A x\|:\|x\| \leq 1\} .
$$

Let $C[a, b]_{\mathbb{T}}$ be the vector space of continuous functions $x:[a, b]_{\mathbb{T}} \rightarrow \mathbb{R}^{n}$. Let $\beta>0$ be a fixed real number. It was shown in [17] that $\|\cdot\|_{\beta}$ defined by

$$
\|x\|_{\beta}:=\sup \left\{\frac{\|x(t)\|}{e_{\beta}(t, a)}: t \in[a, b]_{\mathbb{T}}\right\}
$$

is a norm on $C[a, b]_{\mathbb{T}}$ and is equivalent to the norm $\|\cdot\|_{0}$ given by

$$
\|x\|_{0}:=\sup \left\{\|x(t)\|: t \in[a, b]_{\mathbb{T}}\right\} .
$$

Furthermore, it was concluded in $\left[\mathbf{1 7}\right.$, Lemma 3.3] that $\left(C[a, b]_{\mathbb{T}},\|x\|_{\beta}\right)$ is a Banach space.

Denote by $d_{\beta}$ the metric induced by the norm $\|\cdot\|_{\beta}$, that is for $\zeta, \varphi \in C[a, b]_{\mathbb{T}}$

$$
d_{\beta}(\zeta, \varphi):=\|\zeta-\varphi\|_{\beta}=\sup \left\{\frac{\|\zeta(t)-\varphi(t)\|}{e_{\beta}(t, a)}: t \in[a, b]_{\mathbb{T}}\right\} .
$$

Of course, $\left(C[a, b]_{\mathbb{T}}, d_{\beta}\right)$ is a complete metric space. 
Next, we show that equation (11) has a unique solution on $[s, \infty)_{\pi^{\kappa}}$ satisfying $x(s)=x_{0}$.

2.2. Existence theorem. In order to obtain a contraction mapping we need to invert equation (11). Hence, the following result is essential since it enables us to change the order of integration on a triangular region.

Lemma 7. A differentiable function $x(t)$ satisfies (11) and the initial condition $x(s)=x_{0}$ if and only if $x(t)$ solves

$$
x(t)=x_{0}+\int_{s}^{t}\left[A(u)+\int_{\sigma(u)}^{t} B(v, u) x(u) \Delta v\right] x(u) \Delta u+\int_{s}^{t} f(u) \Delta u .
$$

Proof. Integrating (11) from $s$ to $t$ we obtain

$$
x(t)=x_{0}+\int_{s}^{t} A(u) x(u) \Delta u+\int_{s}^{t} \Delta v \int_{s}^{v} B(v, u) x(u) \Delta u+\int_{s}^{t} f(u) \Delta u .
$$

Changing the order of integration (see [1, Lemma 2.1]) we get (13). Conversely, one may show by using Theorem 4 that a differentiable function $x(t)$ solving (13) satisfies (11) and $x(s)=x_{0}$. The proof is complete.

For a fixed $T \in \mathbb{T}^{\kappa}$ define the space

$$
C_{x_{0}}[s, T]_{\mathbb{T}}:=\left\{\zeta \in C[s, T]_{\mathbb{T}}: \zeta(s)=x_{0}\right\} .
$$

Evidently, $C_{x_{0}}[s, T]_{\mathbb{T}}$ is a closed subset of $C[a, b]_{\mathbb{T}}$. Hence, $C_{x_{0}}[s, T]_{\mathbb{T}}$ is complete when it is endowed with the metric induced on $C_{x_{0}}[s, T]_{\mathbb{T}}$ by $d_{\beta}$. Using right-hand side of the equation (13) we can construct a mapping on $C_{x_{0}}[s, T]_{\mathbb{T}}$. Define the mapping $Q$ by

$$
Q \zeta(t):=x_{0}+\int_{s}^{t}\left[A(u)+\int_{\sigma(u)}^{t} B(v, u) \Delta v\right] \zeta(u) \Delta u+\int_{s}^{t} f(u) \Delta u
$$

for all $\zeta \in C_{x_{0}}[s, T]_{\mathbb{T}}$. We derive the next result from continuity of $f, A$ and $B$.

Lemma 8. For each $\zeta \in C_{x_{0}}[s, T]_{\mathbb{T}}$, the function $Q \zeta$ is continuous on $[s, T]_{\mathbb{T}}$ and $Q \zeta(s)=x_{0}$. That is, $Q$ is a mapping from $C_{x_{0}}[s, T]_{\mathbb{\pi}}$ into $C_{x_{0}}[s, T]_{\mathbb{T}}$.

Hereafter, we show that $Q$ is a contraction on $C_{x_{0}}[s, T]_{\mathbb{T}}$. For any $\zeta, \varphi \in C_{x_{0}}[s, T]_{\mathbb{T}}$,

$$
\|Q \zeta(t)-Q \varphi(t)\| \leq \int_{s}^{t}\left[|A(u)|+\int_{\sigma(u)}^{t}|B(v, u)| \Delta v\right]\|\zeta(u)-\varphi(u)\| \Delta u .
$$

Since $A(t)$ and $B(t, u)$ are continuous for $s \leq u \leq t \leq T$, there is a real number $\beta>1$ such that

$$
|A(u)|+\int_{\sigma(u)}^{t}|B(v, u)| \Delta v \leq \beta-1 .
$$

For such a $\beta,(15)$ and the inequality

$$
e^{-\beta(t-s)} \leq e_{\beta}(t, s)^{-1}
$$


(see Lemma 3) imply

$$
\|Q \zeta(t)-Q \varphi(t)\| \leq(\beta-1) \int_{s}^{t}\|\zeta(u)-\varphi(u)\| \Delta u
$$

and therefore,

$$
\begin{aligned}
\frac{\|Q \zeta(t)-Q \varphi(t)\|}{e_{\beta}(t, s)} & \leq(\beta-1) \int_{s}^{t} \frac{\|\zeta(u)-\varphi(u)\|}{e_{\beta}(t, u) e_{\beta}(u, s)} \Delta u \\
& \leq \frac{(\beta-1)}{\beta}\|\zeta-\varphi\|_{\beta} \int_{s}^{t} \frac{\beta}{e_{\beta}(t, u)} \Delta u \\
& =\frac{(\beta-1)}{\beta}\|\zeta-\varphi\|_{\beta} \int_{s}^{t} e_{\beta}^{\Delta_{u}}(u, t) \Delta u \\
& =\frac{(\beta-1)}{\beta}\|\zeta-\varphi\|_{\beta}\left\{1-\frac{1}{e_{\beta}(t, s)}\right\} \\
& \leq \frac{(\beta-1)}{\beta}\|\zeta-\varphi\|_{\beta}\left\{1-\frac{1}{e^{\beta(t-s)}}\right\} \\
& \leq \frac{(\beta-1)}{\beta}\|\zeta-\varphi\|_{\beta} .
\end{aligned}
$$

This and Lemma 8 show that $Q$ is a contraction on $C_{x_{0}}[s, T]_{\mathbb{T}}$. By the Banach contraction mapping principle $Q$ has a fixed point in $C_{x_{0}}[s, T]_{\mathbb{T}}$. Since $T$ was arbitrary, there is unique continuous solution $x$ of $(13)$ on $[s, \infty)_{\pi^{\kappa}}$. This, along with Lemma 7 , yields the following result:

THEOREM 9. (Existence). For a given $x_{0} \in \mathbb{R}^{n}$, there is a unique continuous solution $x$ of $(11)$ on the interval $[s, \infty)_{\Psi^{\kappa}}$ satisfying the initial condition $x(s)=x_{0}$.

3. Joint Continuity. Taking $f(t) \equiv 0$ in (11), for a given $x_{0} \in \mathbb{R}^{m}$ we can conclude by Theorem 9 that there exists a unique continuous solution $x_{s}$ of

$$
x^{\Delta}(t)=A(t) x(t)+\int_{s}^{t} B(t, u) x(u) \Delta u, t \in[s, \infty) \mathbb{\pi}^{\kappa}
$$

satisfying $x_{s}(s)=x_{0}$. Equivalently, by Lemma $7 x_{s}$ is the unique continuous solution of

$$
x(t)=x_{0}+\int_{s}^{t} \xi(t, u) x(u) \Delta u,
$$

where

$$
\xi(t, u)=A(u)+\int_{\sigma(u)}^{t} B(v, u) \Delta v .
$$

Up to now, we have assumed that $s$ is fixed. If we remove this restriction, then $x_{s}(t)$ turns into a function, defined on the set $\Omega=\left\{(t, s) \in \mathbb{T}^{\kappa} \times \mathbb{T}^{\kappa}: t_{0} \leq s \leq t<\infty\right\}$ whose value at $\left(t_{1}, s_{1}\right) \in \Omega$ is $x_{s_{1}}\left(t_{1}\right)$. 
Definition 10. For a given $x_{0} \in \mathbb{R}^{n}$, let $x$ denote the function with domain $\Omega$ whose value value at $(t, s)$ is

$$
x(t, s):=x_{s}(t)
$$

where $x_{s}$ is the unique solution of $(16)$ on $[s, \infty) \rrbracket^{\kappa}$ satisfying $x_{s}(s)=x_{0}$.

Hereafter, we will show that $x$ is jointly continuous in $(t, s)$.

THEOREM 11. The function $x(t, s)$ defined by (19) is continuous on $\Omega$.

Proof. Let us extend the domain $\Omega$ of $x$ to $\left[t_{0}, \infty\right) \mathbb{\pi} \times\left[t_{0}, \infty\right) \mathbb{t}$ by assuming that $x(t, s)=x_{0}$ whenever $\sigma(s)>t$. For any $T>t_{0}$, consider $x(t, s)$ on $\left[t_{0}, T\right]_{\mathbb{T}} \times\left[t_{0}, T\right]_{\mathbb{T}}$. We will first prove that $x(t, s)$ is uniform continuous in $s$ for each $t \in\left[t_{0}, T\right]_{\mathbb{T}}$. For a fixed $s \in\left[t_{0}, T\right]_{\mathbb{T}}$ and for $t \in[s, T]_{\mathbb{T}}$, we get from equation (17) that

$$
\begin{aligned}
|x(t, s)| & \leq\left|x_{0}\right|+\int_{s}^{t}|\xi(t, u)||x(u, s)| \Delta u \\
& \leq\left|x_{0}\right|+\int_{s}^{t}\left[|A(u)|+\int_{\sigma(u)}^{t}|\xi(v, u)| \Delta v\right]|x(u, s)| \Delta u \\
& \leq\left|x_{0}\right|+k \int_{s}^{t}|x(u, s)| \Delta u,
\end{aligned}
$$

where $k>0$ is a constant chosen so that

$$
|\xi(v, u)| \leq|A(u)|+\int_{\sigma(u)}^{t}|\xi(v, u)| \Delta v \leq k
$$

for $(t, u) \in \Omega_{T}$, where

$$
\Omega_{T}:=\Omega \cap\left(\left[t_{0}, T\right]_{\mathbb{V}} \times\left[t_{0}, T\right]_{\mathbb{T}}\right) .
$$

By Gronwall's inequality (Lemma 5) we obtain

$$
|x(t, s)| \leq\left|x_{0}\right| e_{k}(t, s) \text { for }(t, s) \in \Omega_{T} .
$$

Using [3, Remark 2] we arrive at

$$
|x(t, s)| \leq\left|x_{0}\right| e_{k}(t, s) \leq\left|x_{0}\right| \exp (k(t-s))
$$

for $(t, s) \in \Omega_{T}$. Since $|x(t, s)|=\left|x_{0}\right|$ for $\sigma(t)>t$, the inequality

$$
|x(t, s)| \leq\left|x_{0}\right| \exp \left(k\left(T-t_{0}\right)\right)
$$

holds for all $(t, s) \in\left[t_{0}, T\right]_{\mathbb{T}} \times\left[t_{0}, T\right]_{\mathbb{T}}$. Hereafter, we will use (22) to show that for every $\varepsilon>0$ there exists a $\delta>0$ such that $\left|s_{1}-s_{2}\right|<\delta$ implies

$$
\left|x\left(t, s_{1}\right)-x\left(t, s_{2}\right)\right|<\varepsilon
$$

for all $s_{1}, s_{2} \in\left[t_{0}, T\right]_{\mathbb{T}}$ and all $t \in\left[t_{0}, T\right]_{\mathbb{T}}$. Then the proof will follow from Theorem 6 . For convenience, let us suppose that $s_{2}>s_{1}$. Evidently,

$$
\left|x\left(t, s_{1}\right)-x\left(t, s_{2}\right)\right|=0
$$


as $x(t, s)=x_{0}$ for $t \in\left[t_{0}, s_{1}\right]_{\mathbb{T}}$. For $t \in\left(s_{1}, s_{2}\right]_{\mathbb{T}},(17)$ and (21) yield

$$
\begin{aligned}
\left|x\left(t, s_{1}\right)-x\left(t, s_{2}\right)\right| & =\left|x\left(t, s_{1}\right)-x_{0}\right| \\
& \leq \int_{s_{1}}^{t}|\xi(t, u)|\left|x\left(u, s_{1}\right)\right| \Delta u \\
& \leq k \int_{s_{1}}^{s_{2}}\left|x\left(u, s_{1}\right)\right| \Delta u .
\end{aligned}
$$

This along with (22) implies that for $t \in\left(s_{1}, s_{2}\right]_{\mathbb{T}}$

$$
\begin{aligned}
\left|x\left(t, s_{1}\right)-x\left(t, s_{2}\right)\right| & \leq k \int_{s_{1}}^{s_{2}}\left|x_{0}\right| \exp \left(k\left(T-t_{0}\right)\right) \Delta u \\
& =k\left|x_{0}\right|\left(s_{2}-s_{1}\right) \exp \left(k\left(T-t_{0}\right)\right) .
\end{aligned}
$$

For $t \in\left(s_{2}, T\right]_{\mathbb{T}}$, we have

$$
\begin{aligned}
\left|x\left(t, s_{1}\right)-x\left(t, s_{2}\right)\right|= & \left|\int_{s_{1}}^{t} \xi(t, u) x\left(u, s_{1}\right) \Delta u-\int_{s_{2}}^{t} \xi(t, u) x\left(u, s_{2}\right) \Delta u\right| \\
= & \mid \int_{s_{1}}^{t} \xi(t, u) x\left(u, s_{1}\right) \Delta u-\int_{s_{2}}^{t} \xi(t, u) x\left(u, s_{1}\right) \Delta u \\
& +\int_{s_{2}}^{t} \xi(t, u) x\left(u, s_{1}\right) \Delta u-\int_{s_{2}}^{t} \xi(t, u) x\left(u, s_{2}\right) \Delta u \mid \\
\leq & \int_{s_{1}}^{s_{2}}|\xi(t, u)|\left|x\left(u, s_{1}\right)\right| \Delta u \\
& +\int_{s_{2}}^{t}|\xi(t, u)|\left|x\left(u, s_{1}\right)-x\left(u, s_{2}\right)\right| \Delta u .
\end{aligned}
$$

Using (22) again we have

$$
\left|x\left(t, s_{1}\right)-x\left(t, s_{2}\right)\right| \leq k\left|x_{0}\right|\left(s_{2}-s_{1}\right) \exp \left(k\left(T-t_{0}\right)\right)+k \int_{s_{2}}^{t}\left|x\left(u, s_{1}\right)-x\left(u, s_{2}\right)\right| \Delta u
$$

for all $t \in\left(s_{2}, T\right]_{\mathbb{T}}$. Equation (25) shows that the last inequality also holds for $t=s_{2}$. By Gronwall's inequality we have

$$
\left|x\left(t, s_{1}\right)-x\left(t, s_{2}\right)\right| \leq k\left|x_{0}\right|\left(s_{2}-s_{1}\right) \exp \left(k\left(T-t_{0}\right)\right) \exp \left(k\left(t-s_{2}\right)\right)
$$

for all $t \in\left[s_{2}, T\right]_{\mathbb{T}}$. Combining (24-26) we get

$$
\left|x\left(t, s_{1}\right)-x\left(t, s_{2}\right)\right| \leq k\left|x_{0}\right|\left(s_{2}-s_{1}\right) \exp \left(2 k\left(T-t_{0}\right)\right)
$$

for all $t \in\left[t_{0}, T\right]_{\mathbb{T}}$ and $s_{1}, s_{2} \in\left[t_{0}, T\right]_{\mathbb{T}}$ with $s_{2}>s_{1}$. Obviously, (27) is also true for $s_{1}=s_{2}$. We conclude that

$$
\left|x\left(t, s_{1}\right)-x\left(t, s_{2}\right)\right| \leq k\left|x_{0}\right|\left|s_{1}-s_{2}\right| \exp \left(2 k\left(T-t_{0}\right)\right)
$$

for all $t \in\left[t_{0}, T\right]_{\mathbb{T}}$ and $s_{1}, s_{2} \in\left[t_{0}, T\right]_{\mathbb{T}}$, which implies (23). Consequently, $x(t, s)$ is continuous on $\left[t_{0}, T\right]_{\mathbb{T}} \times\left[t_{0}, T\right]_{\mathbb{T}}$ by Theorem 6 . Since $T$ was arbitrary, $x(t, s)$ is continuous on $\left[t_{0}, \infty\right)_{\mathbb{T}} \times\left[t_{0}, \infty\right) \mathbb{t}$, and hence, continuous on $\Omega$. 
4. Principal matrix solution. For a fixed $s \geq t_{0}$ let $S$ denote the set of all solutions $x(t, s)$ of $(16)$ on the interval $[s, \infty)_{\mathbb{T}^{\kappa}}$ corresponding to initial vectors. If $x(t, s)$ and $\tilde{x}(t, s)$ are two such solutions satisfying $x(s, s)=x_{0}$ and $\tilde{x}(s, s)=\tilde{x}_{0}$, then linearity of (16) implies the principle of superposition, namely, that the linear combination $c_{1} x(t, s)+c_{2} \tilde{x}(t, s)$ is a solution of $(16)$ on $[s, \infty) \mathbb{T}^{\kappa}$ for any $c_{1}, c_{2} \in \mathbb{R}$. This shows that the set $S$ is a vector space. Note that $S$ includes all solutions that have their initial values specified at $t=s$, but not those for which an initial function is specified on an interval $\left[s, t_{1}\right]_{\mathbb{T}^{\kappa}}$ for some $t_{1}>s$.

THEOREM 12. For a fixed $s \in\left[t_{0}, \infty\right)_{\llbracket \kappa}$, let $S$ be the set of all solutions of $(16)$ on the interval $[s, \infty)_{\mathbb{\top}^{\kappa}}$ corresponding to initial vectors. Then $S$ is a n-dimensional vector space.

Proof. We have already shown that $S$ is vector space. It remains to be shown that $\operatorname{dim} S=n$. Let $e^{1}, e^{2}, \ldots, e^{n}$ be the standard basis for $\mathbb{R}^{n}$, where $e^{i}$ is the vector with the components $e_{j}^{i}$, given by

$$
e_{j}^{i}= \begin{cases}1 & \text { if } i=j, \\ 0 & \text { if } i \neq j .\end{cases}
$$

By Theorem 9, there are $n$ unique solutions $x^{i}(t, s)$ of (16) on $[s, \infty)_{\llbracket \kappa}$ satisfying $x^{i}(s, s)=e^{i}(i=1,2, \ldots, n)$. Linear independence of $e^{1}, e^{2}, \ldots, e^{n}$ implies that the solutions $x^{i}(t, s)(i=1,2, \ldots, n)$ are linearly independent. We need to show that

$$
S=\operatorname{span}\left\{x^{1}(t, s), \ldots, x^{n}(t, s)\right\} .
$$

Let $x(t, s) \in S$ be any solution of $(16)$ on $[s, \infty) \rrbracket^{\kappa}$ with $x(s, s)=x_{0}$. Let $v_{1}, v_{2}, \ldots, v_{n}$ be any scalars such that

$$
x_{0}=\sum_{i=1}^{n} v_{i} e^{i} .
$$

By linearity of (16), the linear combination

$$
\eta(t, s)=\sum_{i=1}^{n} v_{i} x^{i}(t, s)
$$

is a solution of (16). Since $\eta(s, s)=x_{0}$, uniqueness part of Theorem 9 implies

$$
x(t, s)=\sum_{i=1}^{n} v_{i} x^{i}(t, s)
$$

and (29). The proof is complete.

Hereafter, we shall denote by $Z(t, s)$ the $n \times n$ matrix function,

$$
Z(t, s):=\left[x^{1}(t, s), x^{2}(t, s), \ldots, x^{n}(t, s)\right],
$$

where the columns $x^{i}(t, s)(i=1,2, \ldots, n)$ are the vectors constituting the basis defined in the proof of Theorem 12. Combining (31) and (32) we get

$$
x(t, s)=Z(t, s) x_{0}
$$


Since $x^{i}(s, s)=e^{i}$ for $i=1,2, \ldots, n$, we have

$$
Z(s, s)=I,
$$

where $I$ denotes the $n \times n$ identity matrix.

REMARK 13. Linearly independence of solutions $\left\{x^{1}(t, s), x^{2}(t, s), \ldots, x^{n}(t, s)\right\}$ for a

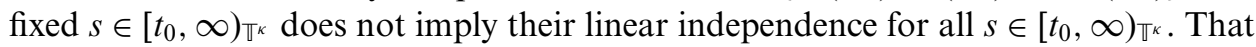
is, $\operatorname{det} Z(t, s)$ may vanish for some $(t, s) \in \Omega$.

DEFINITION 14. The principal matrix solution of (16) is the $n \times n$ matrix function $Z(t, s)$ defined by (32).

Theorem 11 implies that each of the columns $x^{i}(t, s)(i=1,2, \ldots, n)$ of $Z(t, s)$ are continuous on $\Omega$. Hence, we arrive at the following result.

THEOREM 15. The principal matrix solution $Z(t, s)$, defined in (32), is continuous on $\Omega$.

Since the $i$ th column of $Z(t, s)$ is the unique solution of (16) whose value at $t=s$ is $e^{i}, Z(t, s)$ is the unique matrix solution of the initial value problem,

$$
\begin{gathered}
Z^{\Delta_{t}}(t, s)=A(t) Z(t, s)+\int_{s}^{t} B(t, u) Z(u, s) \Delta u, \\
Z(s, s)=I
\end{gathered}
$$

for $(t, s) \in \Omega$. Equivalently, it is the unique matrix solution of

$$
Z(t, s)=I+\int_{s}^{t}\left[A(u)+\int_{\sigma(u)}^{t} B(v, u) \Delta v\right] Z(u, s) \Delta u
$$

by (17) and (18).

5. Variation of parameters formula. Consider the homogeneous equation

$$
x^{\Delta}(t)=A(t) x(t)
$$

where $A$ is an $n \times n$ matrix of continuous functions and $x$ is a column vector of $\Delta$-differentiable functions.

In [4, Theorem 5.8] existence of the unique solution of non-homogeneous equation

$$
x^{\Delta}(t)=A(t) x(t)+f(t)
$$

satisfying $x_{p}(\tau)=x_{0}$ is proven. Moreover, in [4, Theorem 5.24] a variation of parameters formula for the unique solution is given as follows:

$$
x_{p}(t)=e_{A}(t, \tau) x_{0}+\int_{\tau}^{t} e_{A}(t, \sigma(s)) f(s) \Delta s,
$$

where $e_{A}$ is the matrix exponential function (see [4, Definition 5.18]). For more on matrix exponential on time scales, we refer to [4, Section 5] and [19] and references therein). 
If $A$ is regressive (i.e. $I+\mu(t) A(t)$ is invertible for all $t \in \mathbb{T}^{\kappa}$, see [4, Definition $5.5]$ ), then as we did in the proof of Theorem 12, one may show by using the existence theorem [4, Theorem 5.8] that the set of solutions of equation (36) is a vector space of dimension $n$. If $\left(\tilde{x}^{1}, \ldots, \tilde{x}^{n}\right)$ is such a basis, then the matrix $X$ whose columns are the vectors $\tilde{x}^{i}, i=1,2, \ldots, n$, is called a fundamental matrix for equation (36).

In this section, we employ fundamental matrix solution $X(t)$ of homogeneous equation (36) to provide an alternative variation of parameters formula for the unique solution $x_{p}$ of non-homogeneous equation (37) satisfying $x_{p}(\tau)=x_{0}$. We also point out that similar argument cannot be applied to the non-homogeneous equation (11) since the principal matrix solution $Z(t, s)$ of (16) may be singular for some $s$ in $\mathbb{T}$. Finally, we end the section by proposing a variation of parameters formula that does not require any condition for $\operatorname{det} Z(t, s)$.

LEMMA 16. Let $X(t)$ be any fundamental matrix solution of the homogeneous equation (36) and $A$ be a regressive matrix (i.e. $\operatorname{det}(I+\mu(t) A(t)) \neq 0$ ). Then for a given $x_{0} \in \mathbb{R}^{n}$,

1. the solution $x_{h}(t)$ of homogeneous equation (36) satisfying $x_{h}(\tau)=x_{0}\left(\tau \geq t_{0}\right)$ is

$$
x_{h}(t)=X(t) X^{-1}(\tau) x_{0}
$$

2. the solution $x_{p}(t)$ of the non-homogeneous equation (37) satisfying $x_{p}(\tau)=x_{0}$ $\left(\tau \geq t_{0}\right)$ is

$$
x_{p}(t)=X(t) X^{-1}(\tau) x_{0}+\int_{\tau}^{t} X(t) X^{-1}(\sigma(s)) f(s) \Delta s .
$$

Proof. By definition, the columns of a fundamental matrix solution $X(t)$ are linearly independent solutions of (36). Hence, by linearity of (36) for $c \in \mathbb{R}^{n}$ we know that

$$
x_{h}(t)=X(t) c
$$

is a solution of (36). If $x_{h}(\tau)=x_{0}$, then $X(\tau) c=x_{0}$. Since $X(\tau)$ is non-singular, the unique solution for (36) satisfying $x(\tau)=x_{0}$ is $x_{h}(t)=X(t) X^{-1}(\tau) x_{0}$. This is (38). Now, let's look for the solution $x_{p}(t)$ of the non-homogeneous equation (37) of the form

$$
x_{p}(t)=x_{h}(t) u(t)
$$

where $u: \mathbb{T} \rightarrow \mathbb{R}$ is a $\Delta$-differentiable function. Differentiating (40) we get

$$
x_{p}^{\Delta}(t)=x_{h}^{\Delta}(t) u(t)+x_{h}^{\sigma}(t) u^{\Delta}(t)
$$

which along with (37) and (38) implies

$$
f(t)=x_{h}^{\sigma}(t) u^{\Delta}(t)=X(\sigma(t)) X^{-1}(\tau) x_{0} u^{\Delta}(t)
$$

and

$$
x_{0} u^{\Delta}(t)=\left[X(\sigma(t)) X^{-1}(\tau)\right]^{-1} f(t)
$$


The matrix $X(\sigma(t))$ in (41) is non-singular since $X(t)$ is supposed to be a fundamental matrix solution for (36). By (41)

$$
x_{0} u(t)=x_{0}+\int_{\tau}^{t} X(\tau) X^{-1}(\sigma(s)) f(s) \Delta s,
$$

since $u(\tau)=1$ by (40) with $x_{p}(\tau)=x_{h}(\tau)=x_{0}$. From (38) and (40) we obtain (39). The proof is complete.

Notice that (39) turns into (38) when $f=0$.

Using

$$
x_{h}^{\sigma}(t)=x_{h}(t)+\mu(t) x_{h}^{\Delta}(t)=[I+\mu(t) A(t)] x_{h}(t)
$$

and applying the similar procedure in the proof of the preceding lemma we can rewrite (39) as follows:

$$
x_{p}(t)=X(t) X^{-1}(\tau) x_{0}+\int_{\tau}^{t} X(t) X^{-1}(s)[I+\mu(s) A(s)]^{-1} f(s) \Delta s .
$$

REMARK 17. For the derivation of variation of parameter formulas (39) and (42), the essential element is that $X(\sigma(t))$ is non-singular at each $t \in[\tau, \infty)_{\mathbb{T}^{\kappa}}$ or equivalently the matrix $A$ is regressive (i.e. $\operatorname{det}(I+\mu(t) A(t)) \neq 0$ ) and $X(t)$ is singular at each $t \in$ $[\tau, \infty)_{\mathbb{}}$. Note that in [4, Theorem 5.8] regressivity of the matrix $A$ and $r d$-continuity of $f$ are proposed as sufficient conditions for the existence and uniqueness of the unique solution of equation (37).

For the integro-dynamic equation (16), the counterpart of (38) is (33), which we state in the following.

LEMMA 18. The solution of

$$
x^{\Delta}(t)=A(t) x(t)+\int_{\tau}^{t} B(t, u) x(u) \Delta u
$$

on the interval $[\tau, \infty)_{\mathbb{W} \kappa}$ satisfying $x(\tau)=x_{0}$ is

$$
x(t)=Z(t, \tau) x_{0}
$$

where $Z(t, \tau)$ is the principal matrix solution of $(43)$.

If $B=0$ (zero matrix), then (38) and (44) and uniqueness of solutions imply that

$$
Z(t, \tau)=X(t) X^{-1}(\tau)
$$

In this case, (39) turns into

$$
x(t)=Z(t, \tau) x_{0}+\int_{\tau}^{t} Z(t, \sigma(s)) f(s) \Delta s .
$$

Evidently, (45) turns into (38) when $f=0$.

Up to now, we obtained the variation of parameters formula (45) for nonhomogeneous equation (37) by using the solution (38) of the homogeneous equation 
(36). This may lead us to look for a variation of parameters formula for the nonhomogeneous equation (11) by using the principal matrix solution $Z(t, s)$ of the homogeneous equation (16). However, unlike the fundamental matrix solution $X(t)$ of equation (36) (see Remark 17), the principal matrix solution $Z(t, s)$ for equation (16) may be singular at some points as we have stated in Remark 13. To see this in the special case $\mathbb{T}=\mathbb{R}$, one may refer to $[6, \mathrm{p}$. 86]. This shows that we cannot derive a formula for the non-homogeneous equation (11) as we did in Lemma 16 .

The next theorem provides the variation of parameters formula (47), which satisfies (46) even though $Z(t, s)$ is singular for some $(t, s) \in \Omega$.

THEOREM 19. (Variation of parameters). The solution of

$$
x^{\Delta}(t)=A(t) x(t)+\int_{\tau}^{t} B(t, u) x(u) \Delta u+f(t), \quad \tau>t_{0}
$$

on $[\tau, \infty)_{\varpi^{\kappa}}$ satisfying the initial condition $x(\tau)=x_{0}$ is

$$
x(t)=Z(t, \tau) x_{0}+\int_{\tau}^{t} Z(t, \sigma(s)) f(s) \Delta s,
$$

where $Z(t, s)$ is the principal matrix solution of

$$
Z^{\Delta_{t}}(t, s)=A(t) Z(t, s)+\int_{s}^{t} B(t, u) Z(u, s) \Delta u .
$$

Proof. By Theorem 9 there is a unique solution $x(t)$ of $(46)$ on $[\tau, \infty) \mathbb{}$ such that $x(\tau)=x_{0}$. Let us show that

$$
H(t):=Z(t, \tau) x_{0}+\int_{\tau}^{t} Z(t, \sigma(s)) f(s) \Delta s
$$

solves (46). Define $Z(t, s)=I$ for $\sigma(s)>t$. Then $Z(t, s)$ is continuous on $\left[t_{0}, \infty\right) \pi \times$ $\left[t_{0}, \infty\right)_{\mathbb{T}}$ by Theorem 15 . This and (35) show the continuity of $Z^{\Delta_{t}}(t, s)$ on $\left[t_{0}, \infty\right)_{\mathbb{\pi}^{\kappa}} \times$ $\left[t_{0}, \infty\right)_{\pi^{\kappa}}$. From Theorem 4 we conclude that the integral term in (48) is differentiable and that

$$
\begin{aligned}
H^{\Delta}(t)= & Z^{\Delta}(t, \tau) x_{0}+Z(\sigma(t), \sigma(t)) f(t)+\int_{\tau}^{t} Z^{\Delta_{t}}(t, \sigma(s)) f(s) \Delta s \\
= & {\left[A(t) Z(t, \tau)+\int_{\tau}^{t} B(t, u) Z(u, \tau) \Delta u\right] x_{0}+I f(t) } \\
& +\int_{\tau}^{t}\left[A(t) Z(t, \sigma(s))+\int_{\sigma(s)}^{t} B(t, u) Z(u, \sigma(s)) \Delta u\right] f(s) \Delta s \\
= & A(t)\left[Z(t, \tau) x_{0}+\int_{\tau}^{t} Z(t, \sigma(s)) f(s) \Delta s\right]+\left[\int_{\tau}^{t} B(t, u) Z(u, \tau) \Delta u\right] x_{0} \\
& +f(t)+\int_{\tau}^{t}\left[\int_{\tau}^{u} B(t, u) Z(u, \sigma(s)) f(s) \Delta s\right] \Delta u \\
= & A(t) H(t)+\int_{\tau}^{t} B(t, u) H(u) \Delta u+f(t),
\end{aligned}
$$


where we change the order of integration by using [1, Lemma 2.1]. Thus, the function $H$ defined by (48) is a solution of (46) on $[\tau, \infty)_{\mathbb{T}^{\kappa}}$. By $(48), H(\tau)=x_{0}$. Hence, $x(t)=H(t)$ on $[\tau, \infty)_{\mathbb{J}^{\kappa}}$ by uniqueness theorem. The proof is complete.

COROLlary 20. Let $\varphi \in C\left[t_{0}, \tau\right]_{\mathbb{T}}$ for any $\tau>t_{0}$. The solution of

$$
x^{\Delta}(t)=A(t) x(t)+\int_{t_{0}}^{t} B(t, u) x(u) \Delta u+f(t)
$$

on $[\tau, \infty)_{\mathbb{J}^{\kappa}}$ satisfying the condition $x(t)=\varphi(t)$ for $t \in\left[t_{0}, \tau\right)_{\mathbb{\varpi}^{\kappa}}$ is

$$
\begin{aligned}
x(t)= & Z(t, \tau) \varphi(t)+\int_{\tau}^{t} Z(t, \sigma(s)) f(s) \Delta s \\
& +\int_{\tau}^{t} Z(t, \sigma(s))\left\{\int_{t_{0}}^{\tau} B(s, u) \varphi(u) \Delta u\right\} \Delta s .
\end{aligned}
$$

Proof. Since $x \equiv \varphi$ on $\left[t_{0}, \tau\right]_{\mathbb{T}}$, we can rewrite (49) as follows:

$$
x^{\Delta}(t)=A(t) x(t)+\int_{\tau}^{t} B(t, u) x(u) \Delta u+g(t)
$$

where

$$
g(t):=f(t)+\int_{t_{0}}^{\tau} B(t, u) \varphi(u) \Delta u .
$$

By Theorem 9, equation (51) has a unique solution $x$ that is continuous on $[\tau, \infty)_{\rrbracket^{k}}$ such that $x(\tau)=\varphi(\tau)$. By Theorem 19 the solution is

$$
x(t)=Z(t, \tau) \varphi(\tau)+\int_{\tau}^{t} Z(t, \sigma(s)) g(s) \Delta s,
$$

which is (50). This completes the proof.

\section{REFERENCES}

1. M. Adivar and Y. N. Raffoul, Existence of resolvent for Volterra integral equations on time scales, Bull. Aust. Math. Soc. 82(1) (2010), 139-155.

2. L. C. Becker, Principal matrix solution and variation of parameters for a Volterra integro-differential equation and its adjoint, E. J. Qual. Theory Differ. Equ. 14 (2006), 1-22.

3. M. Bohner, Some oscillation criteria for first-order delay dynamic equations, Far East J. Appl. Math. 18(3) (2005), 289-304.

4. M. Bohner and A. Peterson, Dynamic equations on time scales, in An introduction with applications (Birkhäuser Boston, Boston, MA, 2001).

5. M. Bohner and A. Peterson, Advances in dynamic equations on time scales (Birkhäuser Boston, Boston, MA, 2003).

6. T. A. Burton, Stability and periodic solutions of ordinary and functional differential equations (Dover Publications, Mineola, NY, 2005).

7. T. A. Burton, Fixed points, Volterra equations, and Becker's resolvent, Acta Math. Hungar. 108(3) (2005), 261-281.

8. T. A. Burton, Integral equations, Volterra equations, and the remarkable resolvent: Contractions, E. J. Qualitative Theory Differ. Equ. 2(3) (2006), 1-17.

9. S. Elaydi, Periodicity and stability of linear Volterra difference systems, J. Math. Anal. Appl. 181 (1994), 483-492. 
10. S. Elaydi and V. Kocic, Global stability of a nonlinear Volterra difference equations, Differ. Equ. Dyn. Syst. 2 (1994), 337-345.

11. P. Eloe, M. Islam and Y. Raffoul, Uniform asymptotic stability in nonlinear Volterra discrete systems, Comput. Math. Appl. (2002, Special issue advances in difference equations IV) 45(6-9) (2003), 1033-1039. 1946).

12. L. M. Graves, The theory of functions of real variables (McGraw-Hill, New York, NY,

13. S. I. Grossman and R. K. Miller, Perturbation theory for Volterra integro-differential systems, J. Differ. Equ. 8 (1970), 457-474.

14. S. Hilger, Analysis on measure chains - a unified approach to continuous and discrete calculus, Results Math. 18 (1990), 18-56.

15. T. M. Khandaker and Y. N. Raffoul, Stability properties of linear Volterra discrete systems with nonlinear perturbation, J. Differ. Equ. Appl. 8(10) (2002), 857-874.

16. T. Kulik and C. C. Tisdell, Volterra integral equations on time scales: Basic qualitative and quantitative results with applications to initial value problems on unbounded domains. Int. J. Differ. Equ. 3(1) (2008), 103-133.

17. C. C. Tisdell and A. Zaidi, Basic qualitative and quantitative results for solutions to nonlinear, dynamic equations on time scales with an application to economic modelling. Nonlinear Anal. 68(11) (2008), 3504-3524.

18. Y. N. Raffoul, Stability in neutral nonlinear differential equations with functional delays using fixed point theory, Math. Comput. Modell. 2008(7-8) (2004), 691-700. $99-106$.

19. A. Zafer, The exponential of a constant matrix on time scales, ANZIAM J. 48 (2006),

20. B. Zhang, Asymptotic stability criteria and integrability properties of the resolvent of Volterra and functional equations, Funkcialaj Ekvacioj 40, (1997), 335-351. 